\title{
Fixed-bed drying of grains: analysis of the structural properties of packed-beds of non-spherical particles
}

\author{
Altino, H.O.N. ${ }^{\text {a }}$; Ferreira, M.C. ${ }^{a^{*}}$

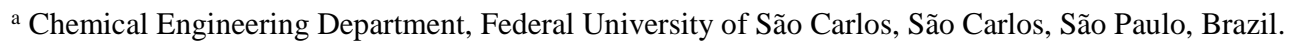 \\ *E-mail of the corresponding author: mariaf@ufscar.br
}

\begin{abstract}
Structural properties of packed-beds of non-spherical biological materials were investigated. The particles tested were oats, lentil, barley and soy, with sphericity ranging from 0.4 to 0.8 . Porous alumina particles with sphericity of 0.98 were also included. The particles dimensions and sphericity were measured under moisture content ranging from the equilibrium up to $24 \%$ (dry basis). Mean voidage, bulk density, permeability and tortuosity of the packed-beds were obtained in the whole moisture range. The data were used as input into a correlation to predict Sherwood numbers under constant air velocity and the results were compared to evaluate the influence of packing characteristics on convective mass transfer.
\end{abstract}

Keywords: Sphericity; Permeability, Tortuosity, Fixed-Bed Dryers. 


\section{Introduction}

It is well-known that variables such as particle shape, size, surface roughness, among others, influence the gas-particle interaction in fixed-bed drying because the packed bed permeability, tortuosity, interparticle contact and residence time are greatly affected by them. For particles of irregular shape and wide size distribution, a great number of packing arrangements can be achieved, leading to changes in the local porosity and contact area that affect the heat and mass transfer rates. Drying is a preservation method widely used for grains, a class of materials that exhibits a widespread variety of shape and sizes. Besides, the dimensions and shape of grains often change throughout drying due to the shrinkage, making the design of drying equipment a challenging task [1]. The influence of the particle properties on fixed-bed drying is useful information for a fundamental analysis of drying processes of grains and seeds.

As a preliminary stage of a study aimed at investigating how the packed-bed characteristics may affect convective fixed-bed drying, a characterization of the packed-bed's structural properties was performed for different biological materials. Particles' characteristic dimensions, volume, equivalent diameter, surface area, sphericity and density were obtained as a function of the moisture content. The bulk density, mean voidage, permeability and tortuosity were obtained for the different packed-beds. The experimental data were used as input to an empirical correlation based on the packed-bed properties to evaluate the mass transfer coefficients and compare the mass transfer potential of each bed.

\section{Materials and Methods}

\subsection{Particulate materials}

The biological materials were selected to cover a wide range of characteristics: soy (Glycine max L.), green lentils (Lens culinaris), barley (Hordeum vulgare L.) and oats (Avena sativa). Nearly spherical porous alumina particles with a diameter of $2.5 \mathrm{~mm}$ were included to serve as a standard material for comparison. The dehydrated materials were purchased from a local market and either humidified or dried to reach specified moisture content $(X)$ (\% d.b.), which were set to $24 \%, 16 \%, 9 \%$ and the equilibrium moisture $\left(X_{e q}\right)$. Humidification was done by placing $150 \mathrm{~g}$ samples in polyethylene plastic packages, adding the necessary amount of deionized water and storing the sealed packages at $4^{\circ} \mathrm{C}$ for $48 \mathrm{~h}$. The drying was performed in a hot-air drying oven (TE-394/1, Tecnal $\left.{ }^{\circledR}\right)$ at $50^{\circ} \mathrm{C}$. 


\subsection{Physicochemical characterization}

Particles were assumed to have a triaxial spheroid shape. The length $\left(L_{p}\right)$, width $\left(W_{p}\right)$ and mean diameter $\left(D_{m}\right)$ of 60 particles were measured by the image analysis technique using the software Image-Pro Plus ${ }^{\circledR}$ 6.0. The thickness $\left(T_{p}\right)$ was measured using a digital caliper. The volume $\left(V_{s}\right)$ and sphericity $(\varphi)$ were calculated according to [2]. The packed-beds' bulk density $\left(\rho_{b}\right)$ was obtained by measuring the weight and volume of the samples, using a digital scale (AS 1000, Marte $^{\circledR}$ ) and a glass measuring cylinder with diameter of $5.0 \times 10^{-2}$ $\mathrm{m}$. Small portions of particles were discharged from a constant height through a funnel into a graduated glass vessel. The vessel was tapped over a flat surface until a constant volume. The apparent density $\left(\rho_{a}\right)$ was estimated by liquid pycnometry with toluene $(99.5 \%$, Dinâmica $\left.{ }^{\circledR}\right)$. The mean voidage $\left(\varepsilon_{b}\right)$ was evaluated from the values of $\rho_{a}$ and $\rho_{b}$ [2]. The measurements were performed in triplicate.

\subsection{Determination of the permeability and tortuosity}

The packed-bed static pressure drops were measured in a cylindrical vessel with diameter $(D)$ of $5.0 \times 10^{-2} \mathrm{~m}$ and height of $29.5 \times 10^{-2} \mathrm{~m}$ (the ratio $D / D_{m}$ varied from $7.0-16.0$ ). The pressure taps were distributed along the vessel height at each $5 \mathrm{~cm}$. The particles were randomly packed according to the same procedure described in section 2.2. The pressures $(P)$ were measured under different air flow rates and at a constant air temperature $\left(27^{\circ} \mathrm{C}\right)$. The air velocity $(U)$ ranged between $0.05-0.58 \mathrm{~m} . \mathrm{s}^{-1}$. The permeability $(\kappa)$ was obtained from a linear regression of Eq. (1), with experimental values of $\Delta P$ and $U$. The tortuosity $(\tau)$ was estimated based on the capillary model, using Eq. (2) [3].

$$
\begin{gathered}
\frac{\Delta P}{U z}=\frac{\mu}{\kappa}+\frac{c}{\sqrt{\kappa}} \rho U \\
\tau=\frac{\varepsilon_{b}^{3} D_{m}^{2} \varphi^{2}}{72 \kappa\left(1-\varepsilon_{b}\right)^{2}}
\end{gathered}
$$

\subsection{Mass transfer coefficients}

To evaluate how the packed-bed structure affect the mass transfer in a hypothetical drying limited by the external resistance, the mass transfer coefficients were estimated from a generalized semi-empirical correlation developed for porous media. As described in [4], the equation assumes that packed-bed structure can be described by a capillary geometry and defines a wall energetic criterion $\left(X e_{w}\right)$. The correlation is given by:

$$
S h=3.66+0.101\left[1+\frac{\pi}{4} \frac{1-\varepsilon_{b}}{\varepsilon_{b}}(\tau-1)\left(1+\frac{\varepsilon_{b}}{\tau^{2}} S c^{\frac{1}{3}} e^{-\frac{11-\varepsilon_{b}}{3 \varepsilon_{b}} \sqrt{\tau Y} X e_{w}^{\frac{1}{8}}}\right)\right] X e_{w}^{\frac{11}{48}} S c^{\frac{1}{3}}
$$


The dimensionless groups $X e_{w}, S h, R e$ and $S c$ were estimated according to [4]. The diffusivity of moisture in the air $\left(D_{A}\right)$ was obtained from [5]. Air physical properties and $D_{A}$ were estimated at ambient temperature $\left(30^{\circ} \mathrm{C}\right)$. The aspect ratio $(Y)$ was obtained from the ratio of particle thickness over particle width, $T_{p} / W_{p}[4]$.

\section{Results and Discussion}

\subsection{Particles characteristics}

The particles' linear dimensions, $\left(L_{p}, W_{p}, T_{p}\right)$, mean diameter $\left(D_{m}\right)$, volume $\left(V_{s}\right)$ and sphericity $(\varphi)$ are shown in Figs. 1(a) to 1(f).
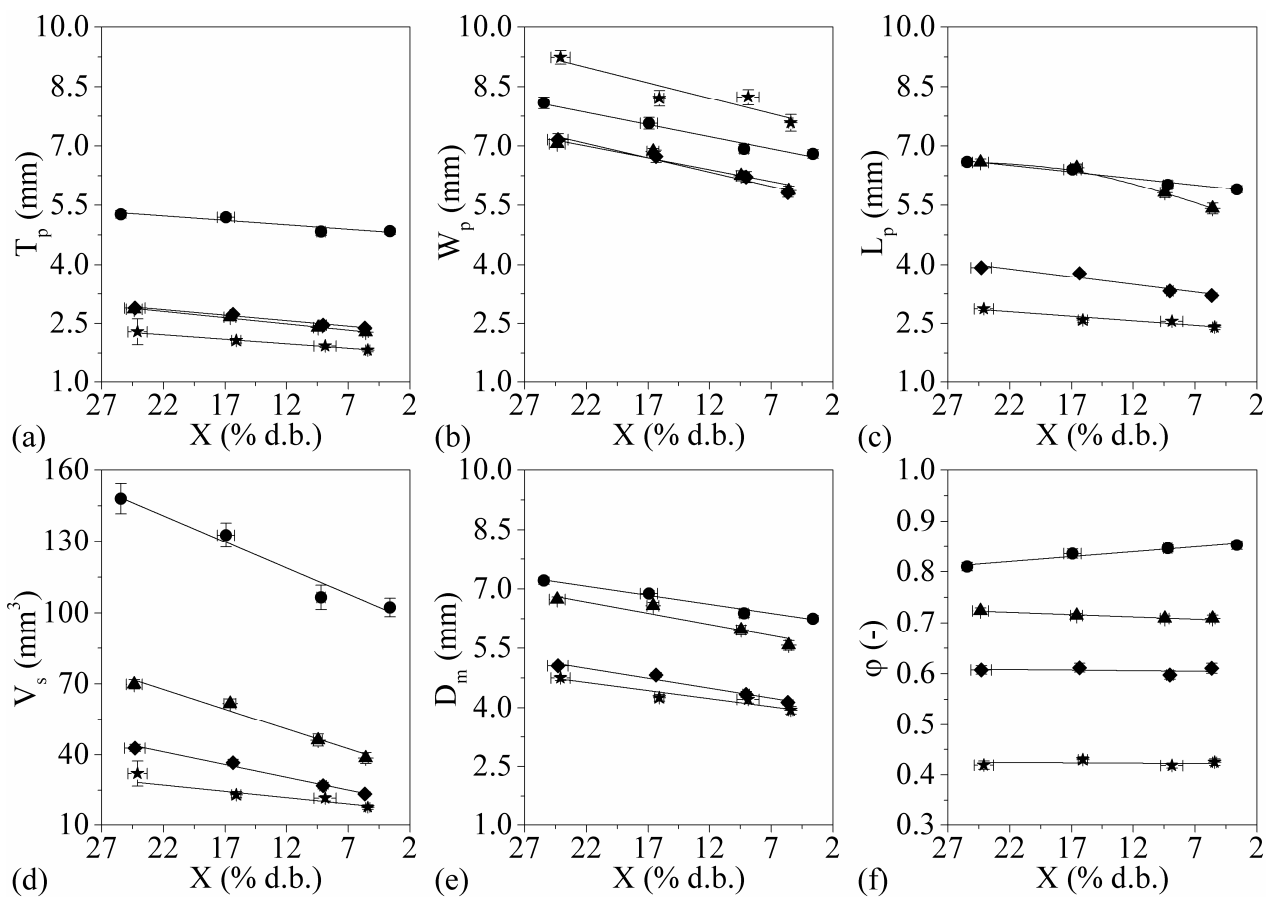

Fig. 1 Particles' dimensions and sphericity as a function of the moisture content; $T_{p}(\mathbf{a}), W_{p}(\mathbf{b}), L_{p}$ $(\mathrm{c}), V_{s}(\mathrm{~d}), D_{m}(\mathrm{e})$ and $\varphi(\mathrm{f})$. Legend: soy $(\bullet)$, lentil $(\Delta)$, barley $(\bullet)$ and oats $(\star)$.

A reduction in $X$ resulted in a linear decrease in the particles' dimensions, owing to the shrinkage phenomenon that appears as a result from an unbalance between the material's inner pressure and the external pressure [6]. Shrinkage is usually observed in food materials with a solid matrix in the rubbery state [6,7]. The particles characteristics of alumina are not shown because its dimensions do not change with moisture variation. The results and 
qualitative behavior of variables in Fig. 1 are consistent with those reported in the literature [8-11]. Although the linear dimensions change, $\varphi$ is not significantly affected by the moisture reduction, as can be observed in Fig. 1(f).

\subsection{Packed-beds structural properties}

The particles' sphericity are, in a decreasing order, 0.98 (alumina), 0.8 (soy), 0.7 (green lentils), 0.6 (barley) and 0.4 (oats). It is worth noting that the particles differ also in size, shape, apparent density and surface roughness. All these variables may affect the packing structure, however the differences cannot be avoided when working with biological particles. The variation of $\rho_{b}$ and $\varepsilon_{b}$ as a function of $\varphi$ are shown in Fig. 2 for $X$ equal to $24 \%$ and for dry particles (equilibrium moisture). The results obtained at $16 \%$ and $9 \%$ stayed between the upper and lower limits and for the sake of concision are not shown in the graphs.
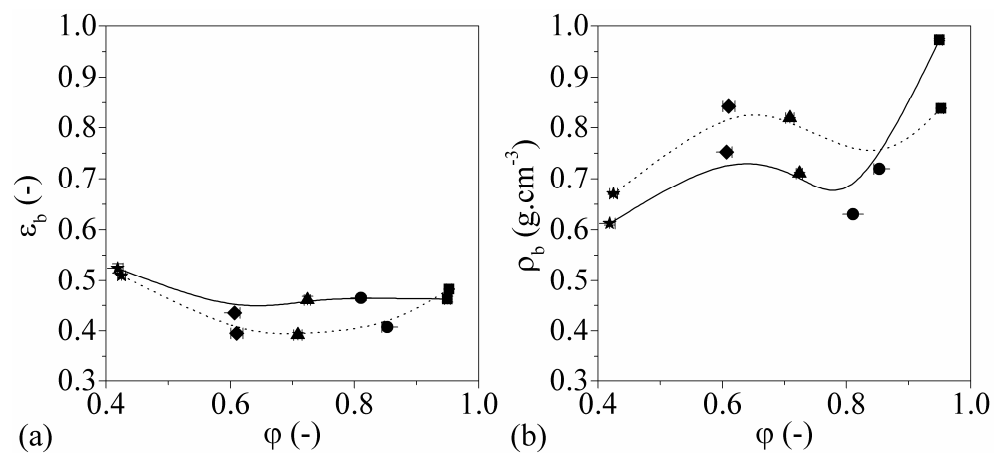

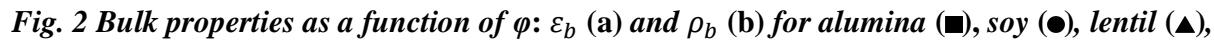
barley ( $\bullet$ ) and oats $(\star)$. Legend: moistures contents of $24 \%(-)$ and $X_{e q}(\cdots \cdots)$.

Fig. 2 (a) shows that at a constant $X, \varepsilon_{b}$ did not vary significantly as $\varphi$ was reduced from 0.98 to 0.60 . Only at $\varphi=0.40$ a slight increase was observed, suggesting that packed-beds of oats tend to produce large pores. However, it is not clear why $\varepsilon_{b}$ was not affected by the moisture content in this particular case. The variation of $\rho_{b}$ with $\varphi$ is complex and does not show a clear trend, as can be seen in Fig. 2(b). At a constant $X, \varepsilon_{b}$ and $\rho_{b}$ depend not only on $\varphi$, but also on additional factors, such as the particles' dimensions and $\rho_{a}$. Additionally, the variation of $D_{m}$ and $\rho_{a}$ with moisture content is different for each particle. Even the particles' surface roughness can be affected by moisture and it is quite difficult identifying the different contributions of each variable in the packed-bed structure. The apparent density of alumina, for instance, decreases as $X$ is reduced, as its volume does not change. On the other hand, the grains shrink and an opposite behavior is observed. Therefore, 
several combined effects are responsible for the patterns observed in Figs. 2(a) and 2(b). The qualitative dependence of $\rho_{b}$ and $\varepsilon_{b}$ on $X$ were similar to those reported by [8-11] for lentil, barley, soy and oats, respectively. A reduction of $X$ caused an increase in $\rho_{b}$ (Fig. 2 (b)) and a decrease in $\varepsilon_{b}$ (Fig. 2 (a)), which is probably associated to the decrease in $D_{m}$ observed as the moisture content is reduced (Fig. 1(e)). For random packing of nonspherical particles, smaller values of $D_{m}$ generate smaller intraparticle void spaces, thus increasing $\rho_{b}$ and decreasing $\varepsilon_{b}$ [12]. Alumina presented an atypical behavior, because $D_{m}$ does not change with $X$ for this particle.

The influence of $X$ on $\kappa$ and $\tau$ is displayed in Fig. 3 as a function of $\varphi$. A decrease in $X$ resulted in lower values of $\kappa$ and higher values of $\tau$. This can be attributed again to the reduction of $D_{m}$ with $X$ due to the shrinkage of grains, and is consistent with the patterns described in Figure 2.
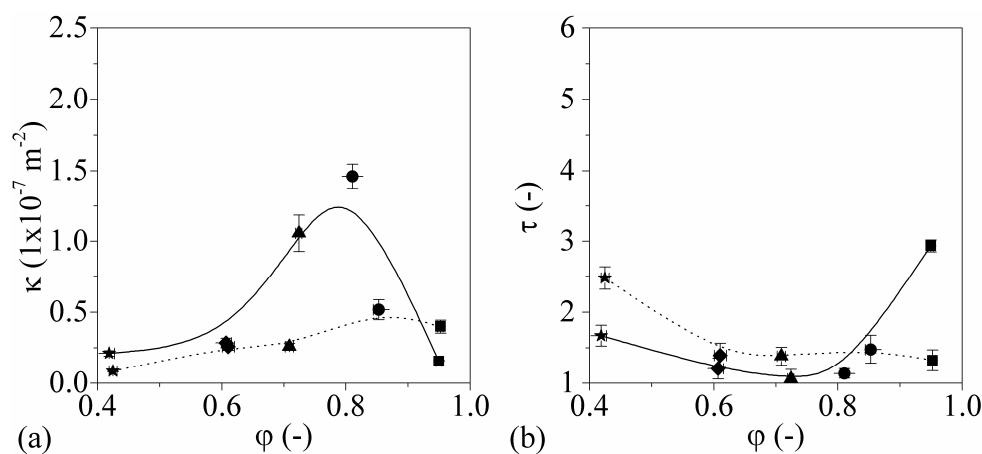

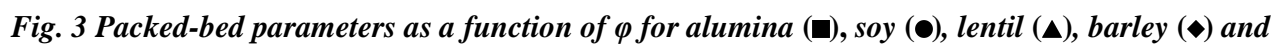
oats $(\star) ; \kappa(a)$ and $\tau(b)$. Legend: moistures contents of $24 \%(-)$ and $X_{e q}(\cdots \cdots)$.

It is also observed in Fig. 3 that, at a constant $X, \kappa$ tends to decrease with the decrease in $\varphi$ and $\tau$ tends to increase. According to the literature [5], deviations from the spherical shape may contribute to reducing the pore diameter. However, for the particles considered here, not only the particle shape but other variables, such as the size and apparent density can affect the packed-beds' structural properties. It can also be observed that the influece of $\varphi$ on $\kappa$ and $\tau$ is much more evident at a high $X$, which is probably related to the dependence of the grains' dimensions on $X$. Once more, an atypical behavior is observed for alumina for the aforementioned reasons. 


\subsection{Mass transfer coefficients}

The values of $R e$ and $S h$ were estimated for the different packed-beds at $U=0.58 \mathrm{~m} . \mathrm{s}^{-1}$ and are shown in Fig. 4 as a function of $\varphi$, for values of $X$ equal to $24 \%, 16 \%, 9 \%$ and $X_{e q}$. Re was calculated based on the estimated capillary diameters.

At $X=24 \%$, it can be observed in Fig.4(a) that $R e$ is practically constant for the packed-beds of different particles, regardless of the value of $\varphi$. Only for alumina a significant increase in $R e$ was observed, which is attributed to the smaller size of this particle. At a low moisture content $(X=9 \%)$, a slight decrease in $R e$ is observed as $\varphi$ increases from 0.4 to 0.7 and it increases again as $\varphi$ is further increased. The variation in this case is much lower than observed at $X=24 \%$. These quite different patterns are justified by the influence of the moisture content on the particles' dimensions. The dependence of $S h$ on $\varphi$ in Fig. 4(b) shows quite similar patterns to those observed in Fig. 4(a), as the convective mass transfer is directly correlated to $R e$. High values of $R e$ favor fluid turbulence, flow eddies and mixing and contribute to increasing mass transfer, as the boundary layer thickness and mass transfer resistance are reduced [13]. Therefore, higher values of $S h$ are observed under higher values of $R e$. Among the biological particles tested, and at a constant $X$, higher $S h$ were observed in the packed-beds of oats, which is the particle of lower sphericity.
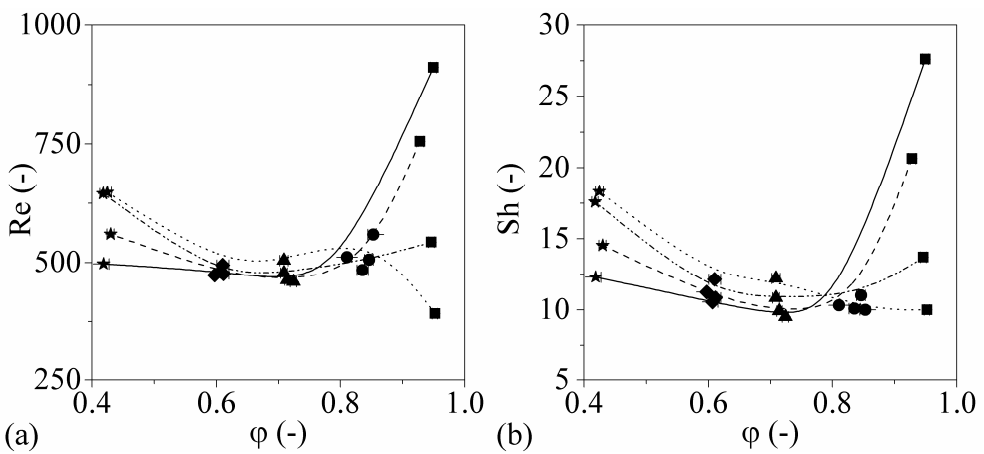

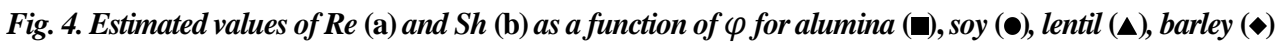
and oats $(\star)$ at $0.58 \mathrm{~m} \cdot \mathrm{s}^{-1}$. Legend: moisture of $24 \%(-), 16 \%(---), 9 \%(-\cdots \cdots)$ and $X_{\text {eq }}(\cdots \cdots)$.

\section{Conclusions}

Based on the experimental data, it was verified that the permeability and tortuosity of packed-beds of soy, oat, lentil and barley were significantly affected by the particles shape and size and by the moisture content. The results suggest that the packed-bed structure may change significantly throughout drying depending on the particle characteristics, as the particles dimensions vary with their moisture content, while their shape practically do not 
change. Because each particle was differently affected by the moisture content and have distinct shape and sizes, no consistent correlation between the structural properties such as mean voidage, bulk density, permeability and tortuosity to the particle characteristics could be identified.

\section{References}

[1] D.B. Brooker, F.W. Bakker-Arkema, W. Hall, Drying and storage of grains and oilseeds, Van Nostrand Reinhold, New York (1992) 450.

[2] N. Mohsenin, Physical properties of plant and animal materials, Gordon and Breach, New York, 1970.

[3] D. Lasseux, F. J. Valdés-Parada. On the developments of Darcy's law to include inertial and slip effects. C. R. Mec. 345 (2017) 660-669

[4] J. Comiti, E. Mauret, M. Renaud, Mass transfer in fixed beds: proposition of a generalized correlation based on an energetic criterion, Chem. Eng. Sci. 55 (2000) 5545-5554.

[5] T.R. Marrero, E.A. Mason, Gaseous diffusion coefficients, J. Phys. Chem. Ref. Data. 1 (1972) 3-118.

[6] L. Mayor, A.M. Sereno, Modelling shrinkage during convective drying of food materials: A review, J. Food Eng. 61 (2004) 373-386.

[7] B.R. Bhandari, T. Howes, Implication of glass transition for the drying and stability of dried foods, J. Food Eng. 40 (1999) 71-79.

[8] S.D. Deshpande, S. Bal, T.P. Ojha, Physical Properties of Soybean, J. Agric. Eng. Res. 56 (1993) 89-98.

[9] E. Isik, Moisture dependent physical and mechanical properties of green laird lentil (Lens culinaris) grains, Pakistan J. Biol. Sci. 10 (2007) 474-480.

[10] C.A. Sologubik, L.A. Campañone, A.M. Pagano, M.C. Gely, Effect of moisture content on some physical properties of barley, Ind. Crops Prod. 43 (2013) 762-767.

[11] A. Shah, F.A. Masoodi, A. Gani, B.A. Ashwar, Geometrical, functional, thermal, and structural properties of oat varieties from temperate region of India, J. Food Sci. Technol. 53 (2016) 18561866.

[12] R.M. German, Particle Paking Characteristics, Metal Powder Industries Federation, Princeton, 1989.

[13] J. Comiti, M. Renaud, Liquid-solid mass transfer in packed beds of parallelepipedal particles: energetic correlation, Chem. Eng. Sci. 46 (1991) 143-154. 